\title{
Study of Thermally Induced Vibration of Non-Homogeneous Trapezoidal Plate with Varying Thickness and Density
}

\author{
Arun Kumar Gupta ${ }^{1, *}$, Pragati Sharma ${ }^{2}$ \\ ${ }^{1}$ Department of Mathematics, M.S. College, Saharanpur, U.P., India \\ ${ }^{2}$ Department of Mathematics, H.C.T.M., Kaithal, Hary ana, India
}

\begin{abstract}
The goal of present investigation is to study the effect of thermal gradient on the vibrations of non-homogeneous trapezoidal plate whose thickness varies parabolically and density varies linearly. Effect of other plate parameters such as non-homogeneity constant, taper constant and aspect ratios have also been studied. C-S-C-S boundary condition with two term deflection is taken into consideration. Rayleigh-Ritz method is used to find the solution of the problem. Results are calculated with great accuracy and presented in tabular form. Comparison of the results with published data has also shown.
\end{abstract}

Keywords Vibration, Trapezoidal Plate, Thermal Gradient, Non-Ho mogeneity, Parabolically Vary ing Thickness

\section{Introduction}

Thermal effect on vibration of non-homogenous plates are of great interest in the field of engineering such as for better designing of gas turbines, jet engine, space craft and nuclear power projects. Such structures are exposed to high intensity heat fluxes and thus material properties undergo significant changes, in particular the thermal effect on the modules of elasticity of the material can not be taken as negligible.

Plates of variable thickness are frequently used in order to economize the plate material or to lighten the plates, especially when it is used in the wings of high-speed and high performance airc rafts. The study of vibration of plates has acquired great importance in the field of research, engineering and space technology. In the engineering we cannot move without considering the effect of vibration because almost machines and engineering structuresexperie nces vibrations. Structures of plates have wide applications in ships, bridges etc. In the aeronautical field, analysis of thermally induced vibrations in non-homogeneous plates of variable thickness has a great interest due to their utility in aircraft wings.

Many analyses show that plate vibrations are based on non-homogeneity of materials. Non-homogeneity can be natural or artificial. Non-homogeneous materials such as

* Corresponding author:

gupta_arunnitin@yahoo.co.in (Arun Kumar Gupta)

Published online at http://journal.sapub.org/ajcam

Copyright (C) 2012 Scientific \& Academic Publishing. All Rights Reserved plywood, delta wood, fibre-reinforced plastic etc, are used in engineering design and technology to strengthen the construction. Study of the effect of vibration cannot be restricted only in the field of science but, our day to day life is also affected by it. Vibration of plates of various shapes, homogeneous or non-homogeneous, orthotropic or isotropic, with or without variation in thickness, have been studied by various authors, with or without considering the effect of temperature.

A large number of researchers have reported about the vibration analysis of different types of plates. So me of them are mentioned here.

Sharma et al.[1] studied the free transverse vibrations of non-homogeneous circular plates of linearly varying thickness. Gupta et al.[2] worked on the vibrations of non-homogeneous rectangular plate of variable thickness in both directions with thermal gradient effect. Gutierrez and Laura [3] calculated the fundamental frequency of vibrating rectangular, non-homogeneous plates. Gupta and Kumar[4] studied the effect of thermal gradient on free vibration of non-homogeneous visco-elastic rectangular plate of parabolically varying thic kness.

Gupta et al.[5] observed the transverse vibration of non-homogeneous orthotropic visco-elastic circular plate of varying parabolic thickness. Lal et al [6] studied the transverse vibrations of non-homogeneous rectangular plates of uniform thickness using boundary characteristic orthogonal polynomials. Gupta et al.[7] observed the effect of non-homogeneity on the free vibrations of orthotropic visco-elastic rectangular plate of parabolic varying thickness. Gupta and Kumar[8] studied the thermal effect on vibration 
of non-homogenous visco-elastic rectangular plate of linear varying thickness. Gupta et al.[9] worked on the vibration of visco-elastic orthotropic parallelogram plate with parabolically thickness variation. El-Sayad and Ghazy[10] studied the Rayleigh-Ritz method for free vibration of midline trapezo idal plates. Gupta and Kumar[11] did the free vibration analysis of non-homogeneous visco-elastic circular plate with varying thickness subjected to thermal gradient. Lal and Dhanpati[12] worked on the transverse vibrations of non-homogeneous orthotropic rectangular plates of variable thickness. Gupta et al.[13] did the vibration analysis of non-homogeneous circular plate of nonlinear thickness variation by differential quadrature method. Chakraverty et al.[14] noticed the effect of non-homogeneity on natural frequencies of vibration of elliptic plates. Chen et al[15] studied on free vibration of non-homogeneous transversely isotropic magneto-electro elastic plates. Gupta et al
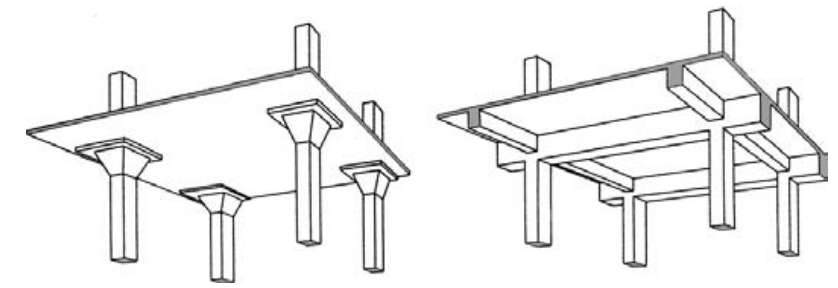

Reinforced concrete slabs in buildings
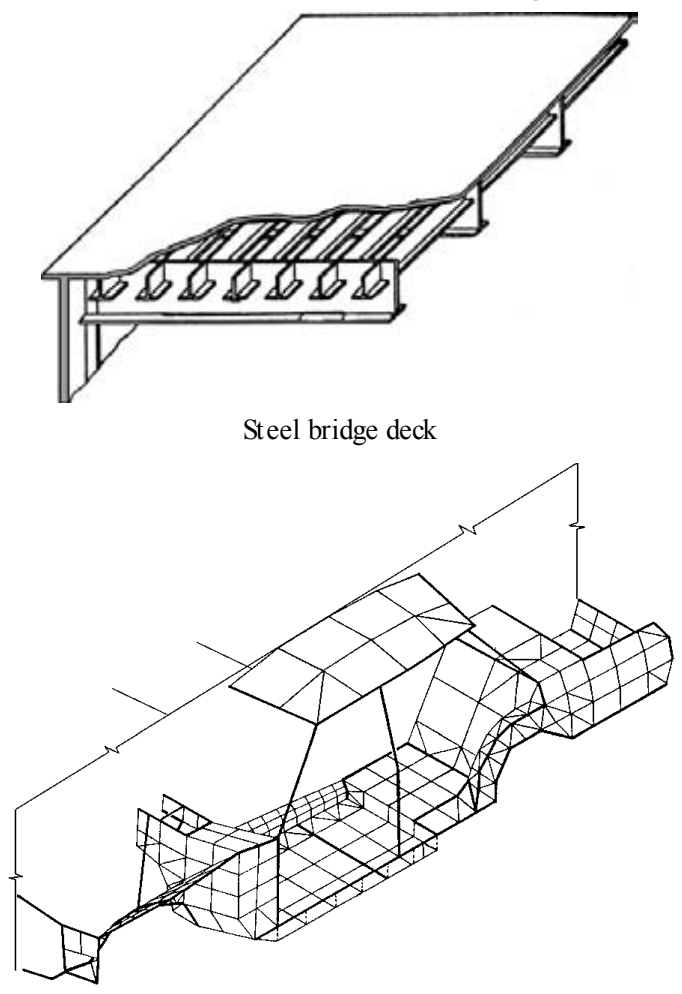

Automobile industry

Figure 1. Use of plates

[16] observedthe effect of non-homogeneity on vibration of orthotropic visco-elastic rectangular plate of linearly varying thickness.
Gupta and Sharma[17] observed the effect of thermal gradient on transverse vibration of non-homogeneous orthotropic trapezoidal plate of parabolically varying thickness. Chen et al[18] studied the free vibration of cantilevered symmetrically laminated thick trapezoidal plates. Bambill et al.[19] studied the transverse vibrations of rectangular, trape zoidal and triangular orthotropic, cantilever plates. Gupta and Sharma[20] observed the thermally induced vibration of orthotropic trapezo idal plate of linearly varying thickness. Gurses et al.[21] analysed the shear deformable laminated composite trapezoidal plates. Kitipornchai et al.[22] discussed a global approach for vibration of thick trapezoidal plates. Gupta and Sharma[23] studied the thermal gradient effect on frequencies of a trapezoidal plate of linearly varying thickness. Gupta and Sharma[24] studied the thermal effect on vibration of non-homogeneous trapezoidal plate of linearly varying thickness.

The authors have so far not come across any paper dealing with parabolically varying thickness and linearly varying density. In the present work the effect of non-homogeneity, taper constant, thermal gradient and aspect ratios has been studied. The frequencies for the first two modes of vibration are obtained for C-S-C-S non-homogeneous trapezoidal plate by Rayleigh-Ritz method. The authenticity and accuracy of numerical results of the present work has been verified with the authors published paper[25]. Results are presented in tabular form. Comparison of results has also been presented.

\section{Theoretical Formulation}

\subsection{Geometry of the Plate}

The plate under consideration is shown in figure 2 which is thin, symmetric and non-homogeneous trapezoidal plate. Here $\mathrm{h}_{0}$ is the maximum plate thickness occurring at the left edge and $\alpha h_{0}$ is the minimum plate thickness occurring at the right edge.

\subsection{Thickness and Density}

The thickness of the plate is parabolic in $\mathrm{xdirection}$ and is of the form

$$
h(\xi)=h_{0}\left[1-(1-\alpha)\left(\xi+\frac{1}{2}\right)^{2}\right]
$$

where $\mathrm{h}_{0}$ is thickness along the edge $\xi=\mathrm{x} / \mathrm{a}=-1 / 2$.

Non-homogeneity of plate arises due to variation in density which is linear in $\mathrm{x}$ direction and is of the form

$$
\rho=\rho_{0}\left[1-\left(1-\alpha_{1}\right)\left(\xi+\frac{1}{2}\right)\right]
$$

It is assumed that the plate considered here is subjected to a steady one-dimensional temperature distribution along the length i.e. in the $\mathrm{x}$ direction, 


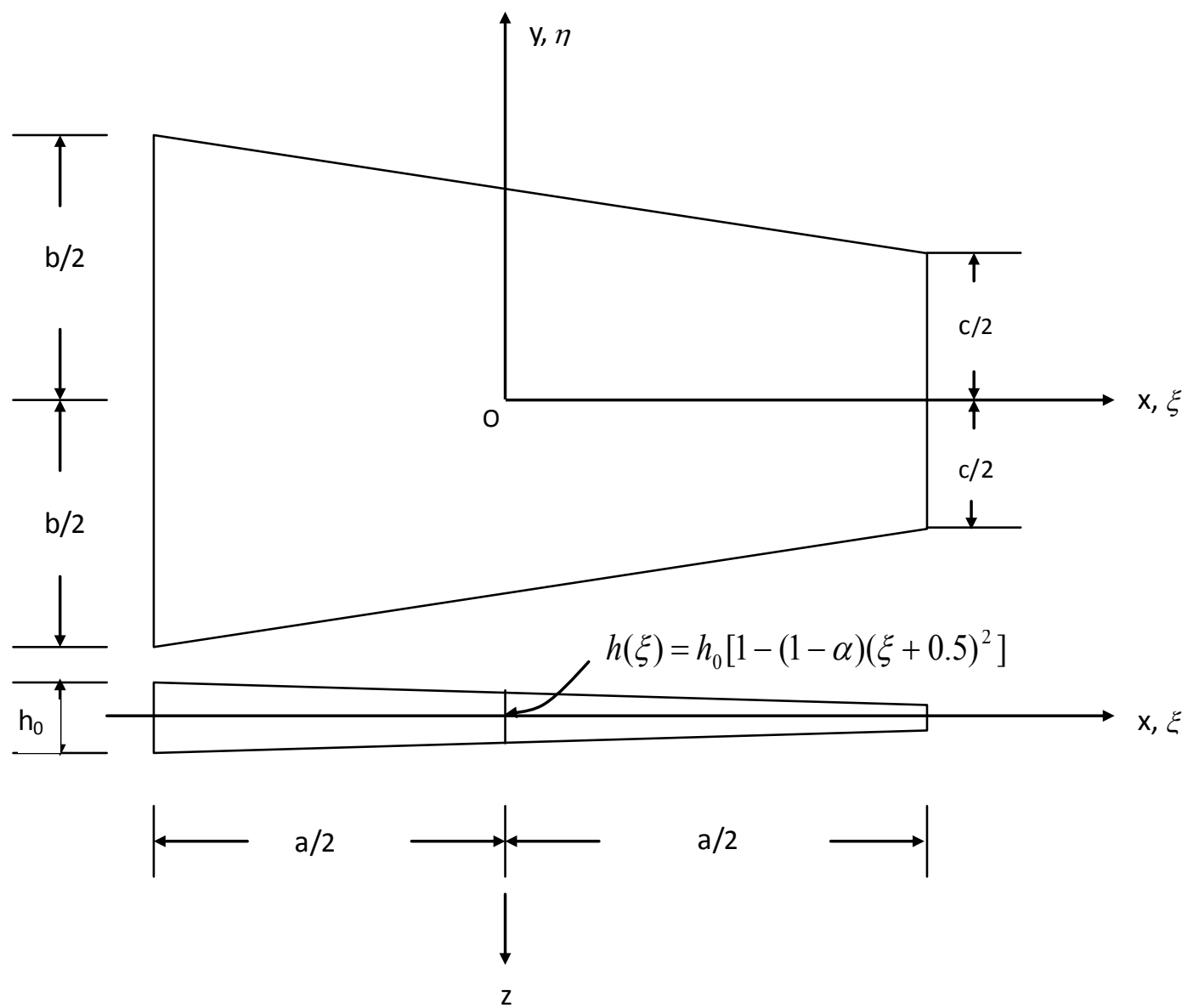

Figure 2. Geometry of trapezoidal plate with variable thickness

\section{Nomenclature of used Symbols}

a length of the plate

b width of the plate at left edge

$\omega$ angular frequency

c width of the plate at right edge

$\mathrm{x}$ longitudinal co-ordinate

$\mathrm{y}$ vertical co-ordinate

$\alpha_{1}$ non-homogeneity constant

$\mathrm{h}$ plate thickness

E Young's modulus

$v$ Poisson's ratio

$\tau$ temperature distribution

$\beta$ thermal gradient

$\alpha$ taper constant

$\rho$ mass density per unit volume of the plate $\xi$ non-dimensional co-ordinate $=\frac{x}{a}$

$\eta \quad$ non-dimensional co-ordinate $=\frac{y}{b}$

$\mathrm{T}$ kinetic energy

$\mathrm{V}$ strain energy

$\lambda$ frequency parameter

$\mathrm{z}$ transverse co-ordinate

w deflection function

$D(\xi)$ flexural rigidity

$\beta$ thermal gradient

$\alpha$ taper constant 


$$
\tau=\tau_{0}\left(\frac{1}{2}-\xi\right)
$$

where $\tau$ denotes the temperature excess above the reference temperature at a distance $\xi=\frac{x}{a}$ and $\tau_{0}$ denotes the temperature excess above the reference temperature at the end $\xi=-\frac{1}{2}$.

For most of the elastic materials, modulus of elasticity is described as

$$
E=E_{0}(1-\gamma \tau)
$$

where $E_{0}$ is the value of Young's modulus along the reference temperature i.e. at $\tau=0$ and $\gamma$ is the slope of the variation of E with $\tau$.

On substituting value of $\tau$ from equation (3) into (4)

$$
E=E_{0}\left(1-\beta\left(\frac{1}{2}-\xi\right)\right)
$$

where $\beta=\gamma \tau_{0} \quad(0 \leq \beta \leq 1)$.

\subsection{Equation of Motion}

The governing differential equation for kinetic energy $\mathrm{T}$ and strain energy $\mathrm{V}$ is given by

$$
\mathrm{T}=\frac{a b}{2} \omega^{2} \int_{A} h(\xi) \rho w^{2} d A
$$

and

$$
\mathrm{V}=\frac{a b}{2} \int_{A} D(\xi)\left\{\left(\frac{1}{a^{2}} \frac{\partial^{2} w}{\partial \xi^{2}}+\frac{1}{b^{2}} \frac{\partial^{2} w}{\partial \eta^{2}}\right)^{2}-2(1-v)\left[\frac{1}{a^{2} b^{2}} \frac{\partial^{2} w}{\partial \xi^{2}} \frac{\partial^{2} w}{\partial \eta^{2}}-\left(\frac{1}{a b} \frac{\partial^{2} w}{\partial \xi \partial \eta}\right)^{2}\right]\right\} d A
$$

in which $D(\xi)$ is the flexural rigid ity of the plate, which is given by

$$
D(\xi)=D_{0}\left[1-(1-\alpha)\left(\xi+\frac{1}{2}\right)^{2}\right]^{3}
$$

Also

$$
D_{0}=\frac{E h_{0}^{3}}{12\left(1-v^{2}\right)}
$$

is the flexural rig idity of the plate at the side $\xi=-\frac{1}{2}, \mathrm{~A}$ is the area of the plate and $\rho$ is the mass density per unit area of the plate.

Using equation (9) and (5) in (8) flexural rigidity is given by

$$
D(\xi)=\frac{E_{0} h_{0}^{3}}{12\left(1-v^{2}\right)}\left[1-(1-\alpha)\left(\xi+\frac{1}{2}\right)^{2}\right]^{3}\left[1-\beta\left(\frac{1}{2}-\xi\right)\right]
$$

Using equation (10) in (7) \& (1), (2) in (6)

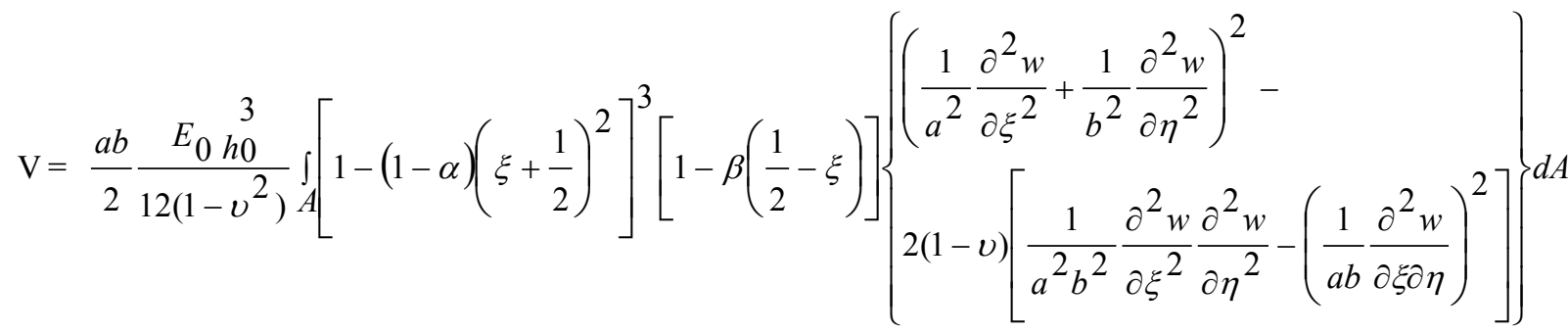

$$
\begin{aligned}
& \mathrm{T}=\frac{a b}{2} \rho_{0} h_{0} \omega^{2} \int_{A}\left[1-(1-\alpha)\left(\xi+\frac{1}{2}\right)^{2}\right]\left[1-\left(1-\alpha_{1}\right)\left(\xi+\frac{1}{2}\right)\right] w^{2} d A
\end{aligned}
$$

\subsection{Deflection Function and Boundary Condition}

A two term deflection function is taken as 


$$
\begin{aligned}
& \left.w=A_{1}\left\{\left(\xi+\frac{1}{2}\right)\left(\xi-\frac{1}{2}\right)\right\}^{2}\left\{\eta-\left(\frac{b-c}{2}\right) \xi+\frac{b+c}{4}\right\} \eta \eta\left(\frac{b-c}{2}\right) \xi-\frac{b+c}{4}\right\} \\
& +A_{2}\left\{\left(\xi+\frac{1}{2}\right)\left(\xi-\frac{1}{2}\right)\right\}^{3}\left\{\eta-\left(\frac{b-c}{2}\right) \xi+\frac{b+c}{4}\right\}^{2}\left\{\eta+\left(\frac{b-c}{2}\right) \xi-\frac{b+c}{4}\right\}^{2}
\end{aligned}
$$

where $\mathrm{A}_{1}$ and $\mathrm{A}_{2}$ are constants to be evaluated. Eq. (13) satisfy boundary conditions and provide a good estimation to the frequency. Clamped-Simply supported-Cla mped-Simply supported plate is taken into consideration as shown in figure.

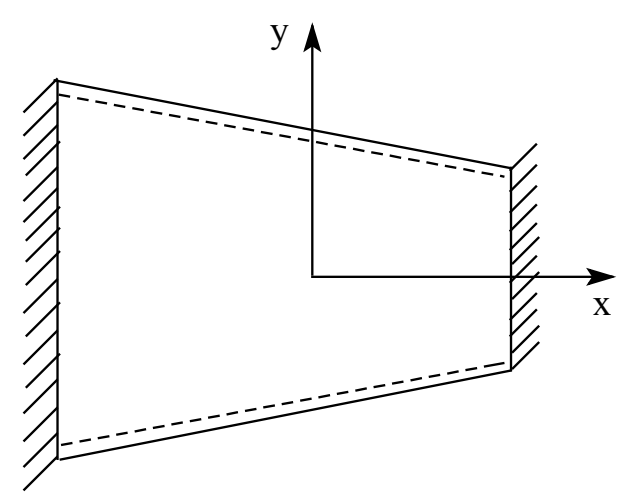

Figure 3. C-S-C-S boundary condition of a trapezoidal plate

Also for the plate considered here boundaries are defined by four straight lines

$$
\begin{aligned}
& \eta=\frac{c}{4 b}-\frac{\xi}{2}+\frac{1}{4}+\frac{c \xi}{2 b} \ldots . \\
& \eta=-\frac{c}{4 b}+\frac{\xi}{2}-\frac{1}{4}-\frac{c \xi}{2 b} . \\
& \xi=-\frac{1}{2} \ldots \ldots \ldots \\
& \xi=\frac{1}{2}
\end{aligned}
$$

\section{Method of Solution}

Rayleigh-Ritz technique is used to find the solution of the problem. According to it maximum kinetic energy must be equal to maximum strain energy, so it is necessary for the problem under consideration that

$$
\delta(V-T)=0
$$

Using equation (14) in (11) and (12)

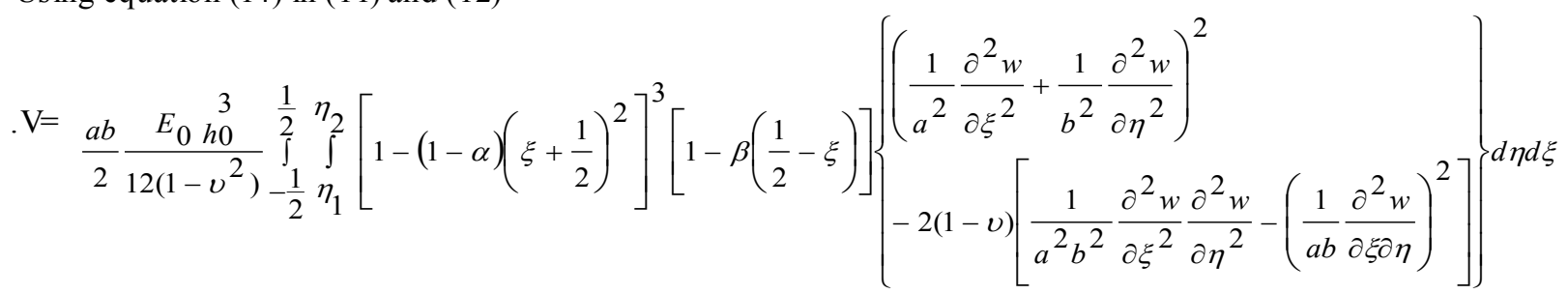

$$
\begin{aligned}
& \mathrm{T}=\frac{a b}{2} \omega^{2} h_{0} \rho_{0} \int_{-\frac{1}{2}}^{\frac{1}{2}} \int_{\eta_{1}}^{\eta_{2}}\left(\left[1-(1-\alpha)\left(\xi+\frac{1}{2}\right)^{2}\right]\left[1-\left(1-\alpha_{1}\right)\left(\xi+\frac{1}{2}\right)\right]\right) w^{2} d \eta d \xi
\end{aligned}
$$

Now (15) becomes

$$
\delta\left(V_{1}-\lambda^{2} T_{1}\right)=0
$$

where 


$$
\begin{aligned}
& V_{1}=\frac{a b}{2} \int_{-\frac{1}{2}}^{\frac{1}{2}} \int_{\eta_{1}}^{\eta_{2}}\left[1-(1-\alpha)\left(\xi+\frac{1}{2}\right)^{2}\right]^{3}\left[1-\beta\left(\frac{1}{2}-\xi\right)\right]\left\{\begin{array}{l}
\left(\frac{1}{a^{2}} \frac{\partial^{2} w}{\partial \xi^{2}}+\frac{1}{b^{2}} \frac{\partial^{2} w}{\partial \eta^{2}}\right)^{2} \\
-2(1-v)\left[\frac{1}{a^{2} b^{2}} \frac{\partial^{2} w}{\partial \xi^{2}} \frac{\partial^{2} w}{\partial \eta^{2}}-\left(\frac{1}{a b} \frac{\partial^{2} w}{\partial \xi \partial \eta}\right)^{2}\right]
\end{array}\right\} d \eta d \xi \\
& T_{1}=\frac{a b}{2} \int_{-\frac{1}{2}}^{\frac{1}{2}} \int_{\eta_{1}}^{\eta_{2}}\left(\left[1-(1-\alpha)\left(\xi+\frac{1}{2}\right)^{2}\right]\left[1-\left(1-\alpha_{1}\right)\left(\xi+\frac{1}{2}\right)\right]\right) w^{2} d \eta d \xi
\end{aligned}
$$

and $\lambda^{2}=\frac{12 \omega^{2} \rho_{0} a^{4}\left(1-v^{2}\right)}{E_{0} h_{0}{ }^{2}}$ is a frequency parameter.

Equation (18) involves the unknown $\mathrm{A}_{1}$ and $\mathrm{A}_{2}$ arising due to the substitution of $w$ from eq (13). These two constants are to be determined from eq. (18), as follows

$$
\begin{aligned}
& \frac{\partial}{\partial A_{1}}\left(V_{1}-\lambda^{2} T_{1}\right)=0 \\
& \frac{\partial}{\partial A_{2}}\left(V_{1}-\lambda^{2} T_{1}\right)=0
\end{aligned}
$$

On simplify ing (21), One gets

$$
b_{m 1} A_{1}+b_{m 2} A_{2}=0, \mathrm{~m}=1,2
$$

where $b_{m 1}, b_{m 2}(m=1,2)$ involve parametric constant and the frequency parameter.

For a non-zero solution, it is desired that co-efficient of eq. (22) must be zero. So one gets the frequency equation as

$$
\left|\begin{array}{ll}
b_{11} & b_{12} \\
b_{21} & b_{22}
\end{array}\right|=0
$$

From eq. (23), one can obtain a quadratic equation in $\lambda^{2}$ from which the two values can found which constitutes first and second mode of vibration.

\section{Results and Discussion}

Frequencies for the first two modes of vibrations are computed for non-homogeneous trapezoidal plate whose thickness varies parabolically and density varies linearly. Different values of non-homogeneity constant $\left(\alpha_{1}\right)$, taper constant $(\alpha)$, thermal gradient $(\beta)$ and aspect ratios $(\mathrm{a} / \mathrm{b}$, $\mathrm{c} / \mathrm{b})$ has been considered. All results are presented in tabular form.

Table 1 and 2: These tables shows the effect of taper constant $\alpha(0.0$ to 1.0$)$ on the frequency parameter $\lambda$ for $\beta=0.0,0.4, \alpha_{1}=0.4,1.0, \mathrm{a} / \mathrm{b}=1.0$ and $\mathrm{c} / \mathrm{b}=0.5,1.0$.

Table 3 and 4: These tables shows the behaviour of the frequency parameter $\lambda$ with thermal gradient $\beta$ ( 0.0 to 1.0 ), for $\alpha=0.0,0.4, \alpha_{1}=0.4,1.0, \mathrm{a} / \mathrm{b}=1.0$ and $\mathrm{c} / \mathrm{b}=0.5$, 1.0 .

Table 5, 6, 7 and 8: These tables show the effects of the frequency parameter $\lambda$ with aspect ratio $\mathrm{c} / \mathrm{b}$ for different combinations of $\alpha$ and $\beta$ as follows.
(i) $\alpha=0.0, \beta=0.0$
(ii) $\alpha=0.0, \beta=0.4$
(iii) $\alpha=0.4, \beta=0.0$
(iv) $\alpha=0.4, \beta=0.4$.

Non-homogeneity constant $\alpha_{1}$ varies from 0.0 to 0.4 , aspect ratio $\mathrm{a} / \mathrm{b}$ varies from 0.75 to 1.0 and aspect ratio $\mathrm{c} / \mathrm{b}$ varies from 0.25 to 1.0 .

Table 9 and 10: In these tables effect of non-homogeneity constant $\alpha_{1}(0.0$ to 1.0$)$ on frequency parameter has been shown. Four combinations of $\alpha$ and $\beta$ (as in table 5 to 8), two values of aspect ratio $\mathrm{c} / \mathrm{b}(0.5,1.0)$ and one value of aspect ratio $\mathrm{a} / \mathrm{b}(1.0)$ has been taken.

In table $\mathbf{1}$ and $\mathbf{2}$ it is observed that the frequency parameter $\lambda$ increases with increasing values of taper constant $\alpha$ for both the modes of vibration. The rate of increase as well as the value of frequency parameter $\lambda$ is higher in second mode in comparison to the first mode.

Further on comparing the results of table $\mathbf{1}$ and $\mathbf{2}$ it is found that on increasing the value of aspect ratio $\mathrm{c} / \mathrm{b}$ fro $\mathrm{m} 0.5$ to 1.0 , frequency parameter decreases for both the modes of vibration.

In table 3 and $\mathbf{4}$ it is seen that frequency parameter $\lambda$ decreases with increasing values of thermal gradient $\beta$ whatever be the values of other plate parameters. The rate of decrease as well as the value of frequency parameter $\lambda$ is higher in second mode in comparis on to the first mode. A lso on increasing aspect ratio $\mathrm{c} / \mathrm{b}$ from 0.5 to 1.0 , frequency parameter decreases for both the modes of vibration.

It is noticed from table 5, 6, 7 and 8 that the frequency parameter $\lambda$ decreases with increasing values of aspect ratio $\mathrm{c} / \mathrm{b}$. Further if we compare table 5 and $6(a / b=0.75)$ it is found that the frequency parameter decreases with increase in non-homogeneity constant $\alpha_{1}$ i.e. from 0.0 to 0.4 . Similar pattern is observed on comparing table 7 and $8(a / b$ $=1.0$ ).

Now if we compare table 5 and $\mathbf{7}$ in which aspect ratio a/b increased from 0.75 to 1.0 and non-homogeneity constant 
$\alpha_{1}$ is 0.0 . It is observed that frequency parameter increases for both the mode ofvibration. Similar pattern is observed on comparing table $\mathbf{6}$ and $\mathbf{8}$ in which non-homogeneity constant $\alpha_{1}$ is 0.4 .

Table 9 and $\mathbf{1 0}$ clearly shows that frequency parameter $\lambda$ decreases with increasing value of non-homogeneity constant $\alpha_{1}$. Also when aspect ratio c/b increased from 0.5 to 1.0 , frequency parameter decreases for both the mode of vibration

Table 1. Frequency parameter $(\lambda)$ for a trapezoidal plate for different values of taper constant ( $\alpha$ ), thermal gradient $(\beta=0.0,0.4)$,

non-homogeneity constant $\left(\alpha_{1}=0.4,1.0\right)$ and aspect ratios $(\mathrm{a} / \mathrm{b}=1.0),(\mathrm{c} / \mathrm{b}=0.5)$

\begin{tabular}{|c|c|c|c|c|c|c|c|c|}
\hline \multirow{2}{*}{$\alpha$} & \multicolumn{9}{|c|}{$\alpha_{1}=0.4$} & \multicolumn{4}{c|}{$\alpha_{1}=1.0$} \\
\cline { 2 - 9 } & \multicolumn{2}{|c|}{$\beta=0.0$} & \multicolumn{2}{|c|}{$\beta=0.4$} & \multicolumn{2}{c|}{$\beta=0.0$} & \multicolumn{2}{c|}{$\beta=0.4$} \\
\cline { 2 - 9 } & First mode & Secondmode & First mode & $\begin{array}{c}\text { Second } \\
\text { mode }\end{array}$ & $\begin{array}{c}\text { First } \\
\text { mode }\end{array}$ & $\begin{array}{c}\text { Second } \\
\text { mode }\end{array}$ & $\begin{array}{c}\text { First } \\
\text { mode }\end{array}$ & $\begin{array}{c}\text { Second } \\
\text { mode }\end{array}$ \\
\hline 0.0 & 40.256534 & 212.145227 & 38.399268 & 197.390839 & $\begin{array}{c}35.065970 \\
* 35.065961\end{array}$ & $\begin{array}{c}182.752281 \\
* 182.746902\end{array}$ & $\begin{array}{c}33.450533 \\
* 33.450525\end{array}$ & $\begin{array}{c}170.030136 \\
* 170.025133\end{array}$ \\
\hline 0.2 & 40.694632 & 217.649163 & 38.690549 & 201.779358 & $\begin{array}{c}35.374329 \\
* 35.374321\end{array}$ & $\begin{array}{c}186.667554 \\
* 186.662068\end{array}$ & $\begin{array}{c}33.634739 \\
* 33.634732\end{array}$ & $\begin{array}{c}173.043975 \\
* 173.038890\end{array}$ \\
\hline 0.4 & 41.315380 & 224.100543 & 39.126969 & 206.984913 & $\begin{array}{c}35.846034 \\
* 35.846026\end{array}$ & $\begin{array}{c}191.470899 \\
* 191.465274\end{array}$ & $\begin{array}{c}33.949773 \\
* 33.949766\end{array}$ & $\begin{array}{c}176.834596 \\
* 176.829402\end{array}$ \\
\hline 0.6 & 41.135120 & 231.429879 & 39.719495 & 212.936786 & $\begin{array}{c}36.494082 \\
* 36.494075\end{array}$ & $\begin{array}{c}197.078225 \\
* 197.072435\end{array}$ & $\begin{array}{c}34.404108 \\
* 34.404101\end{array}$ & $\begin{array}{c}181.318257 \\
* 181.312931\end{array}$ \\
\hline 0.8 & 43.162560 & 239.564593 & 40.474435 & 219.566355 & $\begin{array}{c}37.324937 \\
* 37.324931\end{array}$ & $\begin{array}{c}203.409607 \\
* 203.403628\end{array}$ & $\begin{array}{c}35.002260 \\
* 35.002254\end{array}$ & $\begin{array}{c}186.419448 \\
* 186.413969\end{array}$ \\
\hline 1.0 & 44.399415 & 248.432258 & 41.393677 & 226.808352 & $\begin{array}{c}38.339109 \\
* 38.339103\end{array}$ & $\begin{array}{c}210.390192 \\
* 210.384004\end{array}$ & $\begin{array}{c}35.745014 \\
* 35.745009\end{array}$ & $\begin{array}{c}192.070139 \\
* 192.664490\end{array}$ \\
\hline
\end{tabular}

*Comparison with author's paper[25]

Table 2. Frequency parameter $(\lambda)$ for a trapezoidal plate for different values of taper constant $(\alpha)$, thermal gradient $(\beta=0.0,0.4)$, non-homogeneity constant $\left(\alpha_{1}=0.4,1.0\right)$ and aspect ratios $(\mathrm{a} / \mathrm{b}=1.0),(\mathrm{c} / \mathrm{b}=1.0)$

\begin{tabular}{|c|c|c|c|c|c|c|c|c|}
\hline \multirow{3}{*}{$\alpha$} & \multicolumn{4}{|c|}{$\alpha_{1}=0.4$} & \multicolumn{4}{|c|}{$\alpha_{1}=1.0$} \\
\hline & \multicolumn{2}{|c|}{$\beta=0.0$} & \multicolumn{2}{|c|}{$\beta=0.4$} & \multicolumn{2}{|c|}{$\beta=0.0$} & \multicolumn{2}{|c|}{$\beta=0.4$} \\
\hline & First mode & Secon d mode & First mode & Secon d mode & $\begin{array}{c}\text { First } \\
\text { mode }\end{array}$ & $\begin{array}{l}\text { Second } \\
\text { mode }\end{array}$ & $\begin{array}{l}\text { First } \\
\text { mode }\end{array}$ & $\begin{array}{c}\text { Second } \\
\text { mode }\end{array}$ \\
\hline 0.0 & 27.137212 & 129.818486 & 25.582627 & 120.041241 & $\begin{array}{c}22.991546 \\
* 22.991536\end{array}$ & $\begin{array}{c}110.916591 \\
* 110.910143 \\
\end{array}$ & $\begin{array}{c}21.672240 \\
* 21.672232\end{array}$ & $\begin{array}{r}102.573392 \\
* 102.567428\end{array}$ \\
\hline 0.2 & 27.776268 & 135.027269 & 26.022172 & 124.151877 & $\begin{array}{c}23.459693 \\
* 23.459684 \\
\end{array}$ & $\begin{array}{c}114.739371 \\
* 114.732949 \\
\end{array}$ & $\begin{array}{c}21.976609 \\
* 21.976661 \\
\end{array}$ & $\begin{array}{r}105.505327 \\
* 105.499419 \\
\end{array}$ \\
\hline 0.4 & 28.810372 & 141.525176 & 26.755336 & 129.293254 & $\begin{array}{c}24.265689 \\
* 24.265681 \\
\end{array}$ & $\begin{array}{c}119.698898 \\
* 119.692419 \\
\end{array}$ & $\begin{array}{c}22.533901 \\
* 22.533893 \\
\end{array}$ & $\begin{array}{r}109.357883 \\
* 109.351961 \\
\end{array}$ \\
\hline 0.6 & 30.310361 & 149.380763 & 27.832078 & 135.501410 & $\begin{array}{c}25.465902 \\
* 25.465893 \\
\end{array}$ & $\begin{array}{r}125.829673 \\
* 125.823062 \\
\end{array}$ & $\begin{array}{c}23.383283 \\
* 23.383276 \\
\end{array}$ & $\begin{array}{r}114.140639 \\
* 114.134641 \\
\end{array}$ \\
\hline 0.8 & 32.291094 & 158.567660 & 29.269554 & 142.751267 & $\begin{array}{c}27.070086 \\
* 27.070079\end{array}$ & $\begin{array}{c}133.090534 \\
* 133.083727\end{array}$ & $\begin{array}{c}24.536971 \\
* 24.536965\end{array}$ & $\begin{array}{r}119.815929 \\
* 119.809800\end{array}$ \\
\hline 1.0 & 34.723315 & 169.000947 & 31.056561 & 150.979092 & $\begin{array}{c}29.051609 \\
* 29.051602\end{array}$ & $\begin{array}{r}141.395696 \\
* 141.388637\end{array}$ & $\begin{array}{c}25.983783 \\
* 25.983776\end{array}$ & $\begin{array}{r}126.317599 \\
* 126.311293\end{array}$ \\
\hline
\end{tabular}

*Comparison with author's paper[25] 
Table 3. Frequency parameter $(\lambda)$ for a trapezoidal plate for different values of thermal gradient $\beta$, taper constant $(\alpha=0.0,0.4)$, non-homogeneity constant $\left(\alpha_{1}=0.4,1.0\right)$ and aspect ratios $(\mathrm{a} / \mathrm{b}=1.0),(\mathrm{c} / \mathrm{b}=0.5)$

\begin{tabular}{|c|c|c|c|c|c|c|c|c|}
\hline \multirow{3}{*}{$\beta$} & \multicolumn{4}{|c|}{$\alpha_{1}=0.4$} & \multicolumn{4}{|c|}{$\alpha_{1}=1.0$} \\
\hline & \multicolumn{2}{|c|}{$\alpha=0.0$} & \multicolumn{2}{|c|}{$\alpha=0.4$} & \multicolumn{2}{|c|}{$\alpha=0.0$} & \multicolumn{2}{|c|}{$\alpha=0.4$} \\
\hline & First mode & Secondmode & First mode & $\begin{array}{c}\text { Second } \\
\text { mode }\end{array}$ & $\begin{array}{l}\text { First } \\
\text { mode }\end{array}$ & $\begin{array}{c}\text { Second } \\
\text { mode }\end{array}$ & $\begin{array}{l}\text { First } \\
\text { mode }\end{array}$ & $\begin{array}{c}\text { Second } \\
\text { mode }\end{array}$ \\
\hline 0.0 & 40.256534 & 212.145227 & 41.315380 & 224.100543 & $\begin{array}{c}35.065970 \\
* 35.065961\end{array}$ & $\begin{array}{c}182.752281 \\
* 182.746902\end{array}$ & $\begin{array}{c}35.846034 \\
* 35.846026\end{array}$ & $\begin{array}{c}191.47089 \\
191.465274\end{array}$ \\
\hline 0.2 & 39.340063 & 204.900649 & 40.236848 & 215.712401 & $\begin{array}{c}34.268787 \\
* 34.268778\end{array}$ & $\begin{array}{c}176.505672 \\
* 176.500477\end{array}$ & $\begin{array}{c}34.911436 \\
* 34.911429\end{array}$ & $\begin{array}{c}184.29796 \\
184.292563\end{array}$ \\
\hline 0.4 & 38.399268 & 197.390839 & 39.126969 & 206.984913 & $\begin{array}{c}33.450533 \\
* 33.450525\end{array}$ & $\begin{array}{r}170.030136 \\
* 170.025133\end{array}$ & $\begin{array}{c}33.949773 \\
* 33.949766\end{array}$ & $\begin{array}{c}176.83456 \\
176.829402\end{array}$ \\
\hline 0.6 & 37.431669 & 189.584409 & 37.982515 & 197.873273 & $\begin{array}{c}32.609076 \\
* 32.609068 \\
\end{array}$ & $\begin{array}{c}163.298552 \\
* 163.293747 \\
\end{array}$ & $\begin{array}{c}32.958278 \\
* 33.949766 \\
\end{array}$ & $\begin{array}{c}169.04239 \\
* 169.037405 \\
\end{array}$ \\
\hline 0.8 & 36.434222 & 181.443259 & 36.799556 & 188.321868 & $\begin{array}{c}31.741798 \\
* 31.741790\end{array}$ & $\begin{array}{c}156.277992 \\
* 156.273393\end{array}$ & $\begin{array}{c}31.933586 \\
* 31.933579\end{array}$ & $\begin{array}{c}160.87363 \\
* 160.868909\end{array}$ \\
\hline 1.0 & 35.403079 & 172.920390 & 35.573177 & 178.260238 & $\begin{array}{c}30.845400 \\
* 30.845392\end{array}$ & $\begin{array}{c}148.927826 \\
* 148.923444\end{array}$ & $\begin{array}{c}30.871501 \\
* 30.845392\end{array}$ & $\begin{array}{c}152.26796 \\
* 152.263514\end{array}$ \\
\hline
\end{tabular}

*Comparison with author's paper[25]

Table 4. Frequency parameter $(\lambda)$ for a trapezoidal plate for different values of thermal gradient $\beta$, taper constant $(\alpha=0.0,0.4$ ), non-homogeneity constant $\left(\alpha_{1}=0.4,1.0\right)$ and aspect ratios $(\mathrm{a} / \mathrm{b}=1.0),(\mathrm{c} / \mathrm{b}=1.0)$

\begin{tabular}{|c|c|c|c|c|c|c|c|c|}
\hline \multirow{3}{*}{$\beta$} & \multicolumn{4}{|c|}{$\alpha_{1}=0.4$} & \multicolumn{4}{|c|}{$\alpha_{1}=1.0$} \\
\hline & \multicolumn{2}{|c|}{$\alpha=0.0$} & \multicolumn{2}{|c|}{$\alpha=0.4$} & \multicolumn{2}{|c|}{$\alpha=0.0$} & \multicolumn{2}{|c|}{$\alpha=0.4$} \\
\hline & First mode & Secondmode & $\begin{array}{l}\text { First } \\
\text { mode }\end{array}$ & Secon d mode & $\begin{array}{c}\text { First } \\
\text { mode }\end{array}$ & $\begin{array}{c}\text { Second } \\
\text { mode }\end{array}$ & $\begin{array}{c}\text { First } \\
\text { mode }\end{array}$ & $\begin{array}{c}\begin{array}{c}\text { Second } \\
\text { mode }\end{array} \\
\end{array}$ \\
\hline 0.0 & 27.137212 & 129.818486 & 28.810372 & 141.525176 & $\begin{array}{c}22.991546 \\
* 22.991536 \\
\end{array}$ & $\begin{array}{c}110.916591 \\
* 110.910143 \\
\end{array}$ & $\begin{array}{c}24.265689 \\
* 24.265681 \\
\end{array}$ & $\begin{array}{r}119.698898 \\
* 119.692419 \\
\end{array}$ \\
\hline 0.2 & 26.373790 & 125.024966 & 27.803427 & 135.546939 & $\begin{array}{c}22.343699 \\
* 22.343690\end{array}$ & $\begin{array}{c}106.826042 \\
* 106.819831\end{array}$ & $\begin{array}{c}23.417148 \\
* 23.417140\end{array}$ & $\begin{array}{c}114.644772 \\
* 114.638565\end{array}$ \\
\hline 0.4 & 25.582627 & 120.041241 & 26.755336 & 129.293254 & $\begin{array}{c}21.672240 \\
* 21.672232\end{array}$ & $\begin{array}{c}102.573392 \\
* 102.567423\end{array}$ & $\begin{array}{c}22.533901 \\
* 22.533893\end{array}$ & $\begin{array}{r}109.357883 \\
* 109.351961\end{array}$ \\
\hline 0.6 & 24.759615 & 114.842863 & 25.659917 & 122.722257 & $\begin{array}{c}20.973665 \\
* 20.973657\end{array}$ & $\begin{array}{c}98.137837 \\
* 98.132128\end{array}$ & $\begin{array}{c}21.610729 \\
* 21.610722\end{array}$ & $\begin{array}{c}103.802872 \\
* 103.797250\end{array}$ \\
\hline 0.8 & 23.899346 & 109.399700 & 24.509039 & 115.780313 & $\begin{array}{c}20.243358 \\
* 20.243350\end{array}$ & $\begin{array}{c}93.493737 \\
* 93.488298\end{array}$ & $\begin{array}{c}20.640763 \\
* 20.640756\end{array}$ & $\begin{array}{c}97.934444 \\
* 97.929139\end{array}$ \\
\hline 1.0 & 22.994423 & 103.673916 & 23.291528 & 108.396812 & $\begin{array}{c}19.475003 \\
* 19.474995\end{array}$ & $\begin{array}{c}88.608913 \\
* 88.603757\end{array}$ & $\begin{array}{c}19.614563 \\
* 19.614556\end{array}$ & $\begin{array}{c}91.692977 \\
* 91.688009\end{array}$ \\
\hline
\end{tabular}

*Comparison with author's paper[25] 
Table 5. Frequency parameter $(\lambda)$ for a trapezoidal plate for different combinations of thermal gradient $(\beta)$, taper constant $(\alpha)$ and fixed value of non-homogeneity constant $\left(\alpha_{1}=0.0\right) \&$ aspect ratio $(\mathrm{a} / \mathrm{b}=0.75)$

\begin{tabular}{|c|c|c|c|c|c|c|c|c|}
\hline \multirow{2}{*}{$\mathrm{c} / \mathrm{b}$} & \multicolumn{2}{|c|}{$\alpha=0.0, \beta=0.0$} & \multicolumn{2}{c|}{$\alpha=0.0, \beta=0.4$} & \multicolumn{2}{c|}{$\alpha=0.4, \beta=0.0$} & \multicolumn{2}{c|}{$\alpha=0.4, \beta=0.4$} \\
\cline { 2 - 9 } & $\begin{array}{c}\text { First } \\
\text { mode }\end{array}$ & $\begin{array}{c}\text { Second } \\
\text { mode }\end{array}$ & $\begin{array}{c}\text { First } \\
\text { mode }\end{array}$ & $\begin{array}{c}\text { Second } \\
\text { mode }\end{array}$ & $\begin{array}{c}\text { First } \\
\text { mode }\end{array}$ & $\begin{array}{c}\text { Second } \\
\text { mode }\end{array}$ & $\begin{array}{c}\text { First } \\
\text { mode }\end{array}$ & $\begin{array}{c}\text { Second mode } \\
.25\end{array}$ \\
\hline 50.781420 & 220.637068 & 48.970291 & 208.536396 & 51.363098 & 228.773031 & 49.334318 & 214.886448 \\
\hline .50 & 40.246760 & 173.580809 & 38.626246 & 163.554573 & 40.994438 & 181.617518 & 39.104539 & 169.717936 \\
\hline .75 & 32.961185 & 136.473316 & 31.447791 & 128.221708 & 34.081575 & 144.831410 & 32.205874 & 134.581089 \\
\hline 1.0 & 28.261857 & 110.048152 & 26.787805 & 103.254464 & 29.970990 & 119.141099 & 27.989856 & 110.094693 \\
\hline
\end{tabular}

Table 6. Frequency parameter $(\lambda)$ for a trapezoidal plate for different combinations of thermal gradient ( $\beta$ ), taper constant $(\alpha)$ and fixed value of non-homogeneity constant $\left(\alpha_{1}=0.4\right)$ and aspect rat io $(\mathrm{a} / \mathrm{b}=0.75)$

\begin{tabular}{|c|c|c|c|c|c|c|c|c|}
\hline \multirow[b]{2}{*}{$\mathrm{c} / \mathrm{b}$} & \multicolumn{2}{|c|}{$\alpha=0.0, \beta=0.0$} & \multicolumn{2}{|c|}{$\alpha=0.0, \beta=0.4$} & \multicolumn{2}{|c|}{$\alpha=0.4, \beta=0.0$} & \multicolumn{2}{|c|}{$\alpha=0.4, \beta=0.4$} \\
\hline & First mode & Second mode & First mode & Second mode & First mode & Second mode & First mode & $\begin{array}{l}\text { Second } \\
\text { mode }\end{array}$ \\
\hline .25 & 45.885197 & 194.970902 & 44.254760 & 184.252605 & 46.275063 & 200.752785 & 44.453262 & 188.541551 \\
\hline .50 & 35.727952 & 152.047763 & 34.294279 & 143.244861 & 36.226379 & 157.472069 & 34.562012 & 147.130155 \\
\hline .75 & 28.757349 & 119.005493 & 27.437708 & 111.807045 & 29.552361 & 124.792020 & 27.928466 & 115.949431 \\
\hline 1.0 & 24.278734 & 95.475791 & 23.007455 & 89.601078 & 25.555152 & 102.099955 & 23.863578 & 94.356716 \\
\hline
\end{tabular}

Table 7. Frequency parameter $(\lambda)$ for a trapezoidal plate for different combinations of thermal gradient ( $\beta$ ), taper constant $(\alpha)$ and fixed value of non-homogeneity constant $\left(\alpha_{1}=0.0\right)$ and aspect ratio $(\mathrm{a} / \mathrm{b}=1.0)$

\begin{tabular}{|c|c|c|c|c|c|c|c|c|}
\hline \multirow{2}{*}{$\mathrm{c} / \mathrm{b}$} & \multicolumn{2}{|c|}{$\alpha=0.0, \beta=0.0$} & \multicolumn{2}{|c|}{$\alpha=0.0, \beta=0.4$} & \multicolumn{2}{|c|}{$\alpha=0.4, \beta=0.0$} & \multicolumn{2}{|c|}{$\alpha=0.4, \beta=0.4$} \\
\hline & First mode & Second mode & First mode & Second mode & $\begin{array}{l}\text { First } \\
\text { mode }\end{array}$ & Second mode & First mode & $\begin{array}{l}\text { Second } \\
\text { mode }\end{array}$ \\
\hline .25 & 56.818289 & 301.922778 & 54.464421 & 282.420325 & 58.100262 & 318.155592 & 55.415783 & 295.877891 \\
\hline .50 & 45.347669 & 242.191642 & 43.252070 & 225.365538 & 46.747405 & 258.494530 & 44.267564 & 238.772041 \\
\hline .75 & 37.139193 & 190.213245 & 35.228027 & 176.195015 & 38.835808 & 206.264773 & 36.451631 & 189.317531 \\
\hline 1.0 & 31.590430 & 149.627140 & 29.784542 & 138.340338 & 33.791695 & 165.132010 & 31.382998 & 150.851802 \\
\hline
\end{tabular}

Table 8. Frequency parameter $(\lambda)$ for a trapezoidal plate for different combinations of thermal gradient ( $\beta$ ), taper constant $(\alpha)$ and fixed value of non-homogeneity constant $\left(\alpha_{1}=0.4\right)$ and aspect ratio $(\mathrm{a} / \mathrm{b}=1.0)$

\begin{tabular}{|c|c|c|c|c|c|c|c|c|}
\hline \multirow{2}{*}{$\mathrm{c} / \mathrm{b}$} & \multicolumn{2}{|c|}{$\alpha=0.0, \beta=0.0$} & \multicolumn{2}{c|}{$\alpha=0.0, \beta=0.4$} & \multicolumn{2}{c|}{$\alpha=0.4, \beta=0.0$} & \multicolumn{2}{c|}{$\alpha=0.4, \beta=0.4$} \\
\cline { 2 - 10 } & First mode & Second mode & First mode & Second mode & First mode & Second mode & First mode & $\begin{array}{c}\text { Second } \\
\text { Mode }\end{array}$ \\
\hline .25 & 51.345711 & 266.771196 & 49.222122 & 249.521280 & 52.356914 & 279.123355 & 49.940913 & 259.562574 \\
\hline .50 & 40.256534 & 212.145227 & 38.399268 & 197.390839 & 41.315380 & 224.100543 & 39.126969 & 206.984913 \\
\hline .75 & 32.402442 & 165.867294 & 30.733758 & 153.640922 & 33.676618 & 177.715586 & 31.610679 & 163.106233 \\
\hline 1.0 & 27.137212 & 129.818486 & 25.582627 & 120.041241 & 28.810372 & 141.525176 & 26.755336 & 129.293254 \\
\hline
\end{tabular}


Table 9. Frequency parameter $(\lambda)$ for a trapezoidal plate for different value of non-homogeneity const ant with different combinat ionsofthermal gradient ( $\beta$ ) and taper constant $(\alpha)$ and aspect ratios $(\mathrm{a} / \mathrm{b}=1.0),(\mathrm{c} / \mathrm{b}=0.5)$

\begin{tabular}{|c|c|c|c|c|c|c|c|c|}
\hline \multirow{2}{*}{$\alpha_{1}$} & \multicolumn{2}{|c|}{$\alpha=0.0, \beta=0.0$} & \multicolumn{2}{|c|}{$\alpha=0.0, \beta=0.4$} & \multicolumn{2}{|c|}{$\alpha=0.4, \beta=0.0$} & \multicolumn{2}{|c|}{$\alpha=0.4, \beta=0.4$} \\
\hline & $\begin{array}{l}\text { First } \\
\text { mode }\end{array}$ & $\begin{array}{l}\text { Second } \\
\text { mode }\end{array}$ & $\begin{array}{l}\text { First } \\
\text { mode }\end{array}$ & $\begin{array}{l}\text { Second } \\
\text { mode }\end{array}$ & $\begin{array}{l}\text { First } \\
\text { mode }\end{array}$ & $\begin{array}{l}\text { Second } \\
\text { mode }\end{array}$ & $\begin{array}{l}\text { First } \\
\text { mode }\end{array}$ & $\begin{array}{l}\text { Second } \\
\text { mode }\end{array}$ \\
\hline 0.0 & 45.347669 & 242.191642 & 43.252070 & 225.365538 & 46.747405 & 258.494530 & 44.267564 & 238.772041 \\
\hline 0.2 & 42.575611 & 225.662425 & 40.609931 & 209.975285 & 43.780876 & 239.427415 & 41.46036 & 221.149257 \\
\hline 0.4 & 40.256534 & 212.145227 & 38.399268 & 197.390839 & 41.315380 & 224.100543 & 39.126969 & 206.984913 \\
\hline 0.6 & 38.279188 & 200.817857 & 36.514175 & 186.846016 & 39.224179 & 211.420472 & 37.147611 & 195.267622 \\
\hline 0.8 & 36.567153 & 191.143002 & 34.881886 & 177.840178 & 37.421284 & 200.697373 & 35.440999 & 185.359413 \\
\hline 1.0 & 35.065970 & 182.752281 & 33.450533 & 170.030136 & 35.846034 & 191.470899 & 33.949773 & 176.834596 \\
\hline
\end{tabular}

Table 10. Frequency parameter $(\lambda)$ for a trapezoidal plate for different value of non-homogeneity constant with different combinations of thermal gradient $(\beta)$ and taper constant $(\alpha)$ and aspect ratios $(\mathrm{a} / \mathrm{b}=1.0),(\mathrm{c} / \mathrm{b}=1.0)$

\begin{tabular}{|c|c|c|c|c|c|c|c|c|}
\hline \multirow{2}{*}{$\alpha_{1}$} & \multicolumn{2}{|c|}{$\alpha=0.0, \beta=0.0$} & \multicolumn{2}{|c|}{$\alpha=0.0, \beta=0.4$} & \multicolumn{2}{|c|}{$\alpha=0.4, \beta=0.0$} & \multicolumn{2}{|c|}{$\alpha=0.4, \beta=0.4$} \\
\hline & $\begin{array}{l}\text { First } \\
\text { mode }\end{array}$ & $\begin{array}{l}\text { Second } \\
\text { mode }\end{array}$ & $\begin{array}{l}\text { First } \\
\text { mode }\end{array}$ & Second mode & $\begin{array}{l}\text { First } \\
\text { mode }\end{array}$ & $\begin{array}{l}\text { Second } \\
\text { mode }\end{array}$ & $\begin{array}{l}\text { First } \\
\text { mode }\end{array}$ & $\begin{array}{l}\text { Second } \\
\text { mode }\end{array}$ \\
\hline 0.0 & 31.590430 & 149.627140 & 29.784542 & 138.340338 & 33.791695 & 165.132010 & 31.382998 & 150.851802 \\
\hline 0.2 & 29.111485 & 138.664324 & 27.445292 & 128.213892 & 31.004872 & 151.967636 & 28.793942 & 138.830092 \\
\hline 0.4 & 27.137212 & 129.818486 & 25.582627 & 120.041241 & 28.810372 & 141.525176 & 26.755336 & 129.293254 \\
\hline 0.6 & 25.516778 & 122.482397 & 24.054015 & 113.262403 & 27.024306 & 132.978728 & 25.096244 & 121.487524 \\
\hline 0.8 & 24.155738 & 116.268659 & 22.770243 & 107.519977 & 25.533910 & 125.815731 & 23.711866 & 114.945038 \\
\hline 1.0 & 22.991546 & 110.916591 & 21.672240 & 102.573392 & 24.265689 & 119.698898 & 22.533901 & 109.357883 \\
\hline
\end{tabular}

\section{Confirmation of Results}

The accuracy of the present computations is compared with the published results [25] for C-S-C-S non-homogeneo us trapezoidal plate with non-homogeneity constant $\alpha_{1}=$ 1.0 , aspect ratio $\mathrm{a} / \mathrm{b}=1.0, \mathrm{c} / \mathrm{b}=0.5,1.0$ and thermal gradient $\beta=0.0$ to 1.0 and taper constant $\alpha=0.0$ to 1.0 .

Table 1, 2, 3 and 4 shows a comparis on of the values of frequency parameter obtained in the present problem and published paper of the authors[25]. A very close agreement is seen between the present results and of the published paper in which homogeneous plate with parabolically varying thickness (density is constant) has been considered.

\section{Conclusions}

The main purpose of the present work is to study the effect of thermal gradient on the frequencies of C-S-C-S non-homogeneous trapezoidal plate with other plate parameters as taper constant, non-homogeneity constant and aspect ratios. Thickness of the plate varies parabolically and density varies linearly. Rayleigh-Ritz technique is used to find frequencies for first two modes of vibration. Study shows that frequency parameters increases with increasing value of taper constant and aspect ratio $a / b$ whereas it decreases with increasing value of non-homogeneity constant, thermal grad ient and as pect ratio $\mathrm{c} / \mathrm{b}$.

\section{REFERENCES}

[1] Sharma S, Lal R, Neelam. Free transverse vibrations of non-homogeneous circular plates of linearly varying thickness. Journal of International Academy of Physical Sciences 2011; 15: 187-200.

[2] Gupta AK, Panwar V, Vats RP. Vibrations of non-homogeneous rectangular plate of variable thickness in both directions with thermal gradient effect. Int. J. of Appl. Math and Mech. 2010; 6:19-37.

[3] Gutierrez RH, Laura PAA. Fundamental frequency of 
vibrating rectangular, non-homogeneous plates. Applied Acoustics 1985; 18: 171-180.

[4] Gupta AK, Kumar L. Effect of thermal gradient on free vibration of non-homogeneous visco-elastic rectangu lar plate of parabolically varying thickness. Acta Technica CSAV 2009; 54: 359-374

[5] Gupta AK, Agarwal N, Kaur H. Transverse vibration of non-homogeneous orthotropic visco-elastic circular plate of varying parabolic thickness. Mathematical Methods in the Applied Sciences 2011; 34: 2065-2076.

[6] Lal R, Kumar Y, Gupta US. Transverse vibrations of non-homogeneous rectangular plates of uniform thickness using boundary characteristic orthogonal polynomials. Int. J. Applied Mathematics and Mechanics 2010; 6: 93-109.

[7] Gupta AK, Agarwal N, Gupta DV, Kumar S, Sharma P. Effect of non-homogeneity on the free vibrations of orthotropic visco-elastic rectangular plate of parabolic varying thick-ness. Adv. Studies Theor. Phys. 2010; 4: 467-486.

[8] Gupta AK, Kumar L. Thermal effect on vibration of non-homogenous visco-elastic rectang-ular plate of linear varying thickness. Meccanica 2008; 43: 47-54.

[9] Gupta AK, Kumar A, Gupta DV. Vibration of visco-elastic orthotropic parallelogram plate with parabolically thickness variation. Annals of Faculty Engineering, Hunedoara, International Journal of En gin eering 2012; 10: 61-70.

[10] El-Sayad MA, Ghazy SSA. Rayleigh-Ritz method for free vibration of midline trapezoidal plates. Canadian Journal on Science and Engineering Mathematics 2012; 3: 159-166.

[11] Gupta AK, Kumar L. Free vibration analysis of non-homogeneous visco-elastic circular plate with varying thickness subjected to thermal gradient. Acta Technica CSAV 2011; 56:217-232.

[12] Lal R, Dhanpati. Transverse vibrations of non-homogeneous orthotropic rectangu lar plates of variable thickness. J. Sound and Vibration 2007; 306: 203-214.

[13] Gupta US, Lal R, Sharma S. Vibration analysis of non-homogeneous circular plate of nonlinear thickness variation by differential quadrature method. J. Sound and Vibration 2006; 298: 892-906.
[14] Chakraverty S, Jindal R, Agarwal VK. Effect of non-homogeneity on natural frequencies of vibration of elliptic plates. Meccanica 2007; 42: 585-599.

[15] Chen WQ, Lee KY, Ding HJ. On free vibration of non-homo gen eous transversely isotropicmagneto-electroelas tic plates. J. Sound and Vibration 2005; 279: 237-251.

[16] Gupta AK, Agarwal N, Gupta DV, Kumar M. Study the non-homogeneity effect on vibration of orthotropic visco-elastic rectangular plate of linearly varying thickness. Int. Review of Pure and Applied Mathematics 2010; 6: 235-248.

[17] Gupta AK, Sharma S. Study the effect of thermal gradient on transverse vibration of non-homogeneous orthotropic trapezoidal plate of parabolically varying thickness. Applied Mathematics 2011; 2: 1-10.

[18] Chen CC, Kitipornchai S, Lim CW, Liew KM. Free vibration of cantilevered symmetrically laminated thick trapezoidal plates. Int. J. Mechanical Sciences 1999; 41: 685-702.

[19] Bambill DV, Laura PAA, Rossi RE. Transverse vibrations of rectangu lar, trap ezoidal and trian gular orthotropic, cantilever plates. J. Sound and Vibration 1998; 210: 286-290.

[20] Gupta AK, Sharma S. Thermally induced vibration of orthotropic trapezoidal plate of linearly varying thickness. J. Vibration and Control 2011; 17: 1591-1598.

[21] Gurses M, Civalek O, Ersoy H. Analy sis of shear deformable laminated composite trape-zoidal plates. Materials and Design 2009; 30: 3030-3035.

[22] Kitipornchai S, Xiang Y, Liew KM, Lim MK. A global approach for vibration of thick trap ezoidal plates. Computers and Structures 1994; 53: 83-92.

[23] Gupta AK, Sharma P. Study the thermal gradient effect on frequencies of a trapezoidal plate of linearly varying thickness. Applied Mathematics 2010; 1:356-364.

[24] Gupta AK, Sharma P. Thermal effect on vibration of non-homogeneous trapezoidal plate of linearly varying thickness. Int. J. of Applied Mathematics and Mechanics 2011; 7: 1-17.

[25] Gupta AK, Sharma P. Effect of linear thermal gradient on vibrations of trapezoidal plates whose thickness varies parabolically. J. Vibration and Control 2012; 18: 395-403. 